Document downloaded from:

http://hdl.handle.net/10251/123274

This paper must be cited as:

Luján, JM.; Guardiola, C.; Pla Moreno, B.; Reig, A. (2018). Analytical Optimal Solution to the Energy Management Problem in Series Hybrid Electric Vehicles. IEEE Transactions on Vehicular Technology. 67(8):6803-6813. https://doi.org/10.1109/TVT.2018.2821265

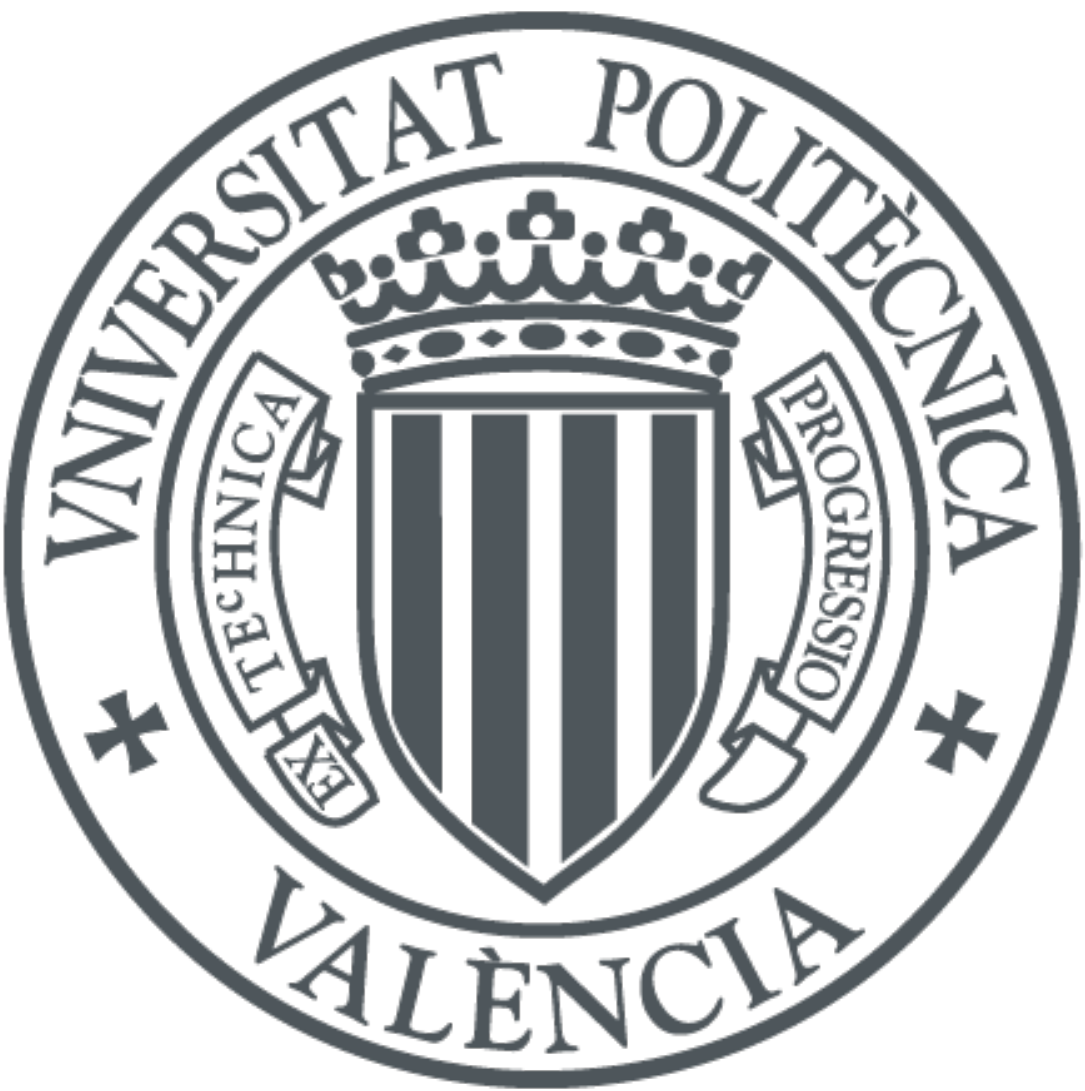

The final publication is available at

http://doi.org/10.1109/TVT.2018.2821265

Copyright Institute of Electrical and Electronics Engineers

Additional Information 


\title{
Analytical optimal solution to the energy management problem in series hybrid electric vehicles
}

\author{
José Manuel Luján, Carlos Guardiola, Benjamín Pla, Alberto Reig* \\ CMT - Motores Térmicos, Universitat Politècnica de València, Valencia (Spain) \\ e-mail: \{jlujan, carguaga, benplamo, alreiber\}@mot.upv.es
}

\begin{abstract}
Optimal solution to the energy management problem in hybrid electric vehicles has been extensively addressed in literature during last decade, especially with the application of dynamic programming, Pontryagin minimum principle or equivalent consumption minimization strategy. However, most of the works consist in finding cycle-specific optimal trajectories which are far from being general control strategies.

The aim of this work is to derive an analytical expression, general and not cycle-specific, for the energy management problem that summarizes the optimal controls for a series hybrid electric vehicle. Starting from a simple definition of the powertrain, an explicit formulation is deducted to minimize fuel consumption based on an analytic analysis of Pontryagin minimum principle. Explicit expressions for control variables and costates are provided. The result is a general control strategy that specifies the optimal generator set usage for a given probability distribution of expected traction demands. This methodology is benchmarked with common methods in literature (dynamic programming and numerical Pontryagin minimum principle) showing near identical results but with strongly reduced computational time. The general form of this control strategy can also be used to analyze the optimal operation range of the engine, which could be useful for designing purposes.
\end{abstract}

\section{Nomenclature}

\section{Introduction}

Energy management problem (EMP) in hybrid electric vehicles (HEV) is a common topic in optimal control literature that has been extensively addressed during the last decade as indicated in [1]. Several methods to solve this problem have been proposed as [2] summarizes. Despite the apparent simplicity of the formulation, the powertrain complexity makes methods incapable of reaching the optimal solution in a general way but dependent on particular driving conditions. Accordingly, many authors base their works on specific driving cycles, bringing the optimal solution to best fit those particular situations [3, 4]. However, those control strategies are
$E_{b} \quad$ Internal battery energy content (stored in the chemicals).

$E_{b, 0} \quad$ Initial internal battery energy content.

$H \quad$ Hamiltonian function.

$J \quad$ Cost index to be minimized (integration of cost function $L$ ).

$L \quad$ Cost function to be minimized.

$P_{b} \quad$ Battery terminals electric power (delivered or absorbed).

$P_{c} \quad$ Battery internal power content stored in the chemicals.

$P_{f} \quad$ Fuel power content.

$P_{g} \quad$ Electrical power delivered by the generator set.

$P_{m} \quad$ Electrical power consumed or delivered by the traction motor.

$P_{f, i d l e}$ Generator set fuel power consumption at idle.

$R \quad$ Battery internal resistance.

$V_{o c} \quad$ Battery open circuit voltage.

$\Psi \quad$ Terminal state constraint function.

* $\quad$ Asterisk denotes an optimal trajectory.

$\eta_{g} \quad$ Generator set fuel to electricity conversion efficiency.

$\lambda(t) \quad$ Costate function as defined at the Pontryagin minimum principle theory.

$\nu \quad$ Costate value (if costate is a constant function).

$\varphi \quad$ Terminal state constraint function adjoined to the cost index.

$\widehat{P}_{g} \quad$ Minimum electric power that the generator set delivers at the optimal solution.

$\widehat{P}_{m} \quad$ Traction motor power threshold for generator set disconnection.

$\widetilde{P}_{m} \quad$ Rearranged traction motor power demand trajectory, required to calculate $\nu$.

$\widetilde{T} \quad$ Total time $P_{m}(t)$ remains over the threshold $\widehat{P}_{m}$.

a Linear coefficient of generator set fuel consumption model.

$f \quad$ State dynamics function.

$g \quad$ Path constraints generic function.

SoC Battery state of charge.

*This work was supported by Ministerio de Economía y Competitividad through Project TRA2016-78717-R. 
only valid for the reference driving trajectory and, consequently, hard to implement in real vehicles since there is no clue on how the optimal solution will perform on a different route. Some examples of cycle-dependent approaches present in literature are dynamic programming (DP) [5-7], Pontryagin minimum principle (PMP) [8-12], equivalent consumption minimization strategy (ECMS) [13-17] or linear programming (LP) [18].

Many different approaches can be found in literature in order to overcome the driving cycle dependency issue and to provide an implementable control algorithm for a real scenario. By far, PMP is the most commonly used for that aim, and two main streams can be found in the existing works: estimation of costate and numerical application of plain PMP theory, and analytic derivation of particular control rules (usually known as "operating modes") from the analysis of PMP necessary conditions.

For the first approach, costate estimation is done in very different ways: extrapolating current usage of the vehicle with a statistic analysis [18-23], using a feedback controller that controls the costate such that battery indicators does not violate constraints and terminal requisites are fulfilled [24-28], assuming the driving cycle as a Markov chain process and predicting a good enough costate [29, 30], using information from an existing optimal solution to the EMP (mostly from DP) to calculate the optimal costate $[12,31,32]$, correlating the observed optimal costate with the driving cycle characteristics [33], estimating the costate by looking on a receding horizon prediction of driving behavior [34], and by using previous geo-located driving information to estimate the most probable optimal costate [35].

The second stream found their works on stating the EMP with PMP theory and, if model is simple enough, optimal controls can be calculated as a function of costate. That brings what it is usually called "operating modes": full throttle, cruising, coasting, maximum regeneration and maximum braking among others. Once operating modes that are optimal under some assumptions according to PMP theory are obtained, the problem is reduced to finding the best combination of those modes to minimize fuel consumption on a cycle. Some works that use this philosophy are [36-38]. Also [39] shows similar concepts, but those operating modes are not deducted from PMP formulation but by observing PMP solutions to particular driving cycles.

Another methodology that has been used in literature to address the EMP is stochastic dynamic programming (SDP) [4, 40], showing promising results but demanding computing requirements that limits its online application.

Most of the above methods are online capable with good performance. However, they need tuning parameters (feedback controllers), sufficient amount of information collected from previous drivings (estimation of costate), or they reduce the EMP to the selection of an operating mode which is only optimum under strong simplifications. Moreover, since they are based on numerical PMP, any change in powertrain parameters (battery aging, different engine performance, etc.) requires a complete recalibration/review of the control strategy.

Approaching these issues, Ambühl et al. presented in [41] a general formulation for a parallel HEV optimal operation. That work was based on a simple parallel HEV model expressed as a set of explicit equations. Starting from those expressions, the optimal control problem is stated and the ECMS and PMP methods are applied. This lets reaching a general control law that is expressed as a function of powertrain parameters and boundary constraints. Several works that also follow a similar philosophy to bring an explicit PMP solution in the case of a fuel cell vehicle [42] or a hybrid truck for battery life control [43].

Following the the above results, this study presents an explicit approach to the EMP. The main contribution of this paper is the methodology to assess the EMP in a completely analytical manner, providing explicit expressions for both control variables and costate calculation. This brings identical results to existing optimal methods but with a fraction of their computational requirements. Also, the explicit dependency on powertrain parameters allows to account for any change (battery aging, etc.) without modifying the control strategy scheme.

This work is based on a non-time dependent HEV model that allows to address the EMP analytically, reaching a general expression for the optimal control policy. This control strategy specifies the optimal generator set usage to minimize fuel consumption as a function of physical powertrain parameters and driver requirements (i.e. traction requirements). As long as the HEV model is non-time dependent, traction requirements can be specified both as a time trajectory or as a probability distribution of the expected traction demands. The only dependency of the optimal control policy on physical parameters allows, on the one hand to deliver a vehicle-specific control straight from the model which can be updated as the powertrain ages and, on the other hand, to optimize powertrain specifications for the particular use of a $\mathrm{HEV}$ based on its optimal control strategy (e.g. to identify typical engine operating zones to assist engine calibration or to fit engine performance for a particular use).

To reach the above targets, the paper is organized as follows: first, a model of a series HEV, the general formulation of a PMP optimization problem and application to the EMP are presented; following, the analytical solution to the EMP is derived from the aforementioned formulation; then, a case study is presented where, on the one hand the analytical solution is benchmarked against DP and numerical PMP in terms of fuel economy and, on the other hand, the EMP solution helps identifying the main engine operation area; finally, results are summarized and several conclusions are drawn.

\section{Problem description}

This section presents the necessary tools to approach the EMP solution: a series HEV model, the general 


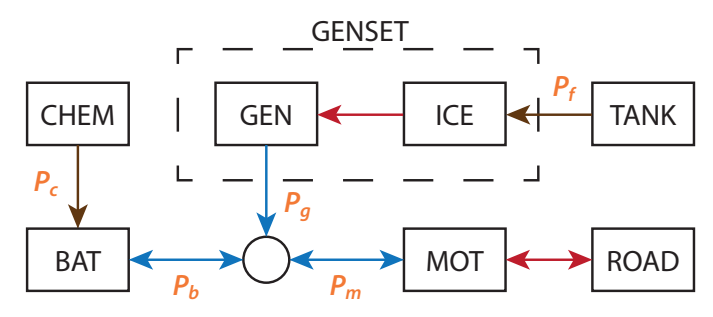

Figure 1: Series HEV powertrain layout scheme. The color of the arrow represents the type of energy that flows through that path: electric (blue), mechanical (red) and chemical (brown). The power flow nomenclature is shown in orange.

formulation of PMP method and its particularization to the EMP. All these tools are strictly kept in an analytical form to reach an explicit EMP solution, avoiding any numerical approximation.

\section{$2.1 \quad$ Model}

A HEV consists of two main energy sources, namely a fuel tank and an electric energy storage system (e.g. battery). The energy demand may be supplied by any of those elements or split into both. In particular, a series HEV consists of a generator set (internal combustion engine coupled to a generator), a battery and a traction motor. The powertrain ultimately satisfies the power requirement with the traction electric motor so the gross power goes through an electric bus, as opposed to parallel architecture where power flows from the crankshaft to the transmission and wheels.

The energy flow, as illustrated in figure 1, starts on one hand at the tank where fuel is sent to the ICE to be converted into mechanical energy. This feeds the generator that powers the main electric bus. On the other hand battery directly feeds the electric bus (mostly through an inverter) at the expense of its internal chemical energy storage. This bus powers the electric traction motor. During decelerations energy regeneration is also possible inverting the path to the battery, increasing its state of charge.

An important advantage of series architecture in modeling is that, since it decouples the ICE rotational speed from vehicle speed, it is possible to run the generator set on any operation point regardless of the current vehicle speed. Therefore, if emissions or engine dynamics are not considered, the ICE operation range can be collapsed to the minimum specific fuel consumption (SFC) curve. Otherwise, full operation map must be considered.

Following, the HEV powertrain model is described. Note that, several simplifications are assumed in order to provide a non-time dependent (i.e. quasi-steady) model as stated in the introduction. A simple but representative model will allow to address the PMP theory explicitly.

\begin{tabular}{ll}
\hline Engine type & Euro V, spark ignited \\
Cylinder configuration & 3 , in-line \\
Engine displacement & 1.2 liter \\
Engine max output & $60 \mathrm{~kW}$ \\
Generator type & $\mathrm{PMSM}$, liquid cooled \\
Generator max output & $50 \mathrm{~kW}$ \\
\hline
\end{tabular}

Table 1: Main engine and generator specifications.

\subsubsection{Generator set}

This work is based on a pre-series range extender unit, consisting of a Euro V spark ignited engine and a $50 \mathrm{~kW}$ generator, whose specifications are at table 1. Figure 2 shows engine, generator and generator set test cell steady state maps. Particularly, the right plot depicts the operation zone resulting from the coupling of the engine and the generator (generator set), where it may be appreciated that the lowest fuel consumption at each electric power output level may be approximated to a linear function (dashed line in black). Accordingly, the generator set is represented with a linear model:

$$
P_{f}= \begin{cases}P_{f, i d l e}+a P_{g} & P_{g}>0 \\ 0 & P_{g}=0\end{cases}
$$

where $P_{f}$ is the fuel power content, $P_{g}$ is the electric power the generator set delivers (the control variable in this work), $P_{f, i d l e}$ is the fuel power consumption when idling, and $a$ is a tunable coefficient. Fuel consumption is zero when generator delivers no power since it is considered switched off in this situation. Throughout this work, the value of these parameters are $P_{f, i d l e}=3.2$ $\mathrm{kW}$ and $a=3$, corresponding to the black dashed line in figure 2. This model entails a generator set efficiency given by:

$$
\eta_{g}=\frac{P_{g}}{P_{f}}=\frac{P_{g}}{P_{f, i d l e}+a P_{g}}
$$

This value converges asymptotically to $1 / a=0.33$ as $P_{g}$ increases.

As shown in figure 2, the correlation between this linear approach and the experimental data is pretty accurate $\left(R^{2}=0.94\right)$. Nevertheless, a more complex model (quadratic, exponential, etc.) could be used if necessary following the same methodology. Anyhow, it must be remarked that, as the reader will find in the following sections, in order to apply explicit PMP theory, first and second derivatives of $P_{f}$ must be calculated. For example, if a quadratic model is used in this case, (27) has no explicit solution unless $P_{f, i d l e}$ and $a$ are set to fixed values (it is a root of a third order polynomial). Therefore, the linear model has been considered sufficient for this work since there is no need to complicate the formulation for representative results.

\subsubsection{Battery}

The battery model consists of an ideal voltage source with a resistance in series. It is a quite simple approach but pretty representative of battery behaviour in a 

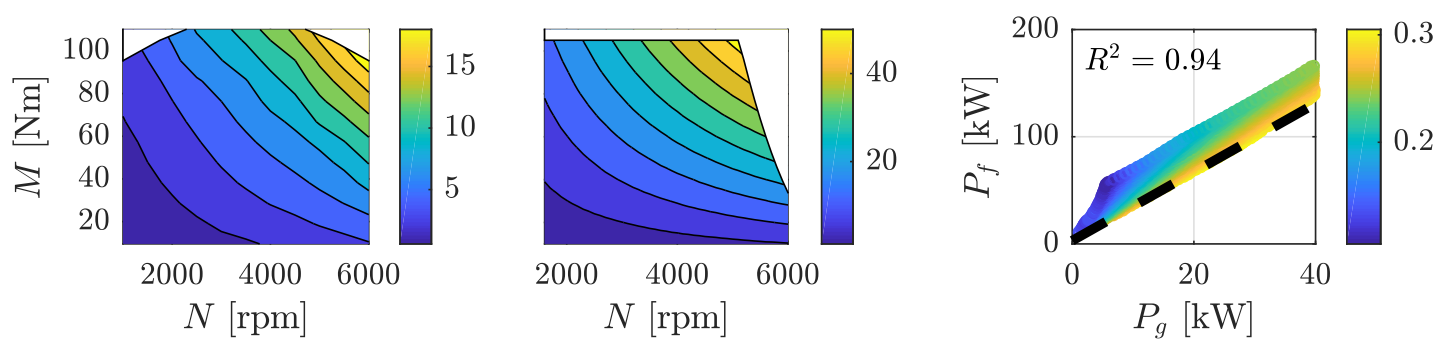

Figure 2: Generator set steady state maps from test cell measuring. The left plot shows the engine fuel consumption map (in $\mathrm{kg} / \mathrm{h}$ ) in terms of speed $(\mathrm{rpm})$ and torque $(\mathrm{Nm})$. The middle plot shows the generator electric power output map (in $\mathrm{kW}$ ) in terms of speed ( $\mathrm{rpm}$ ) and torque $(\mathrm{Nm})$. The right plot shows the efficiency map of the coupling of engine and generator in terms of fuel consumption $(\mathrm{kW})$ and electric power output $(\mathrm{kW})$; the black dashed line is a linear model representing the maximum generator set efficiency.

HEV, as utilized in [44]. Accordingly, the chemical power (function of the internal energy stored in the chemicals), $P_{c}$, that the battery spends to deliver an electric power, $P_{b}$, is:

$$
P_{c}=\frac{V_{o c}}{2 R}\left(V_{o c}-\sqrt{V_{o c}^{2}-4 R P_{b}}\right)
$$

where $V_{o c}$ is the open circuit voltage and $R$ is the battery internal resistance. Note that $P_{b}>0$ discharges the battery according to the sign criterion used in this paper. The energy that remains internally stored in the battery can be calculated from the $P_{c}$ history, so its variation comes defined as:

$$
\dot{E}_{b}=P_{c}
$$

The total energy that remains in the battery is a magnitude that quantifies the current state of the vehicle and, therefore, it is selected as the main state in the presented EMP.

The battery state of charge (SoC) is typically defined as:

$$
\operatorname{SoC}(t)=1-\frac{\int_{0}^{t} i_{b}(t) \mathrm{d} t}{Q_{b, 0}}
$$

with $Q_{b, 0}$ the nominal charge of the battery. Given that $P_{c}=V_{o c} i_{b}$ and $Q_{b, 0}=E_{b, 0} V_{o c}$, the SoC can be calculated from the above problem variables as:

$$
\mathrm{SoC}=1-\frac{E_{b}}{E_{b, 0}}
$$

with $E_{b, 0}$ the nominal energy content of the battery (5.15 MJ is used this model). Note that this expression follows the same definition than the battery state of energy since $V_{o c}$ is constant with SoC.

Additionally, since the voltage drop in the resistance increases with power delivery, there is a maximum amount of power that the battery can achieve, given by:

$$
P_{b, \max }=\frac{V_{o c}^{2}}{4 R}
$$

More detailed models such as those with variable $V_{o c}$ or $R$ as a function of $E_{b}$ could be used to some extent with similar results. However, this dependency on $E_{b}$ introduces time dependency in the model $\left(E_{b}\right.$ is a function of time) which limits the explicit PMP theory application. Particularly, as it will be introduced later to the reader, (24) will no longer hold for a battery model with variable $V_{o c}$ or $R$, and therefore an explicit control cannot be calculated. However, long term effects that introduce a drift in powertrain performance (but which could be considered negligible during a trip) such as battery aging could be taken into account with the current formulation. Due to the explicit dependency on physical parameters, an updated estimation of aged $V_{o c}$ and $R$ can be directly applied to the model and, therefore, the explicit control strategy will immediately account for the new specifications of the powertrain.

\subsubsection{Power split}

Due to the series architecture, the power split at the powertrain must verify that:

$$
P_{b}=P_{m}-P_{g}
$$

with $P_{m}$ the electric power that the traction motor requires which, in this work, is assumed to be a known quantity given by the driver request (as a known trajectory or as an expected probability distribution of traction requirements). As $P_{m}$ is determined, the control does not need a motor neither a vehicle model, which are implicit on the traction requirements; these models are only necessary for simulation. It can also be appreciated that as $P_{m}$ is given and $P_{g}$ is a control variable, the battery power $P_{b}$ is defined by the generator set control strategy.

The powertrain constraints include a minimum and maximum generator set power and rotational speed. The first is introduced by limiting the permitted range of $P_{g}$, while the second is implicitly included in the above fuel consumption model (it must represent a feasible zone of the generator set operation map). The battery also implies a power limitation due to its internal resistance: the voltage drop at the resistance cannot be greater than the battery open circuit voltage, but actually this constraint is overridden by the maximum power the battery can deliver from (7). Finally the traction motor specifications limit the maximum power that might be delivered from/to the vehicle, restricting 
the maximum acceleration that can be performed in a driving cycle.

\subsection{General formulation of Pontryagin Minimum Principle}

An optimal control problem might be formally stated as the minimization of the integral form of a certain cost function $L$ :

$$
J=\varphi(x(T), T)+\int_{0}^{T} L(x(t), u(t), t) d t
$$

where $x$ is the vector of system states and $u \in \mathcal{U}$ is the vector of control actuations, restricted to the subset of permitted actuations $\mathcal{U}$. The main idea of an optimization problem is to find the control trajectory $u(t)$ that minimizes $J$, i.e. $\min _{u}\{J\} . \varphi$ is an arbitrary function that penalizes the cost index to account for any terminal state constraints. Instead of it, a terminal constraint could also be stated as a hard constraint in the form of an additional equation to the problem:

$$
\Psi(x(T), T)=0
$$

where $\Psi$ is another arbitrary function, similar to $\varphi$. This last form is preferred for the present work since it forces the state to adopt a particular value at $t=T$.

The states are driven by the dynamics of the system, defined as a first order differential equation given by a generic function $f$ and with initial values $x(0)=x_{0}$ :

$$
\dot{x}=f(x(t), u(t))
$$

In a general form, the dynamic system is constrained with path constraints with an arbitrary function $g$ as follows:

$$
g(x(t), t) \leq 0
$$

which may also include both upper and lower bounds of state variables.

The optimization problem consists in finding the optimal control trajectory $u^{*}(t)$ that satisfies the above constraints, such that:

$$
J\left(u^{*}(t)\right) \leq J(u(t))
$$

Unfortunately, this kind of problems are difficult to assess in this form since it requires to evaluate the whole time domain to find a solution $u^{*}$. In practical applications, where the full problem is unknown at the time of the optimization, it is not possible to evaluate the integral form of the cost function $L$. However, it is possible to adjoin the system dynamics to $L$ by introducing a vector of costate variables $\lambda$, avoiding the integral form:

$$
\begin{aligned}
H(\lambda(t), x(t), u(t), t)= & L(x(t), u(t), t)+ \\
& \lambda(t) f(x(t), u(t))
\end{aligned}
$$

This construction is the Hamiltonian, as defined by Pontryagin in its Minimum Principle (PMP) [45, 46].
The optimal solution $u^{*}$ and the corresponding optimal trajectory of states, $x^{*}$, and costates, $\lambda^{*}$, must minimize the Hamiltonian:

$$
H\left(\lambda^{*}(t), x^{*}(t), u^{*}(t), t\right) \leq H\left(\lambda^{*}(t), x^{*}(t), u(t), t\right)
$$

The PMP also gives the rest of necessary conditions for optimality of $u^{*}$, namely the terminal constraint, inherited from (10):

$$
\Psi(x(T), T)=0
$$

the trajectory of the costates, also known as costate equations:

$$
\dot{\lambda}(t)=-\frac{\partial H}{\partial x}
$$

and the corresponding terminal constraint for the costates:

$$
\lambda(T)=\left.\frac{\partial \varphi}{\partial x}\right|_{t=T}+\left.\nu \frac{\partial \Psi}{\partial x}\right|_{t=T}
$$

where $\nu$ is a vector of scalars that must be selected in order to satisfy (16). If $x(T)$ is free, $\lambda(T)=0$ as $\varphi$ and $\Psi$ are no longer function of $x$. The PMP theory gives no information on how to select the appropriate $\nu$ value. Several iterative techniques have been proposed in literature to approximate its value, such as shooting method, offline tuning, or heuristics $[1,10,24]$. In section 3.2 a different-non-iterative but direct-method is proposed to analytically extract the optimal $\nu$ value.

\subsection{Pontryagin Minimum Principle ap- plied to the Energy Management Problem}

The HEV energy management problem consists of minimizing the fuel consumption during vehicle's operation along a driving cycle. Therefore, the cost function $L$ corresponds to a function of the fueling rate. Particularly, it is defined as the instantaneous fueling rate expressed in terms of power units (to be coherent with other magnitudes of the problem). Introducing the piecewise expression from (1), the performance index is:

$$
L= \begin{cases}P_{f, i d l e}+a P_{g} & P_{g}>0 \\ 0 & P_{g}=0\end{cases}
$$

According to the proposed HEV model, the only ODE that drives the dynamic system is the gradient in battery energy content-other dynamic effects in the powertrain have been neglected for simplicity and vehicle dynamics are implicitly included in the traction requirement which is known to the problem as it is given by the driver. Consequently, this system is fully defined with just one state: the energy content of the battery, $E_{b}$, given in (4). Therefore, the system dynamics $f$ are conducted by: 


$$
\dot{E}_{b}=f=\frac{V_{o c}}{2 R}\left(V_{o c}-\sqrt{V_{o c}^{2}+4 R\left(P_{g}-P_{m}\right)}\right)
$$

where the right side term is the internal chemical power delivered by the battery from (3) and (8).

The Hamiltonian can be constructed with the above expressions, so (14) is particularized as:

$$
H= \begin{cases}H_{1}=P_{f, i d l e}+a P_{g}+\lambda(t) \dot{E}_{b} & P_{g}>0 \\ H_{2}=\lambda(t) \dot{E}_{b} & P_{g}=0\end{cases}
$$

Additionally, a terminal constraint in battery energy content must be introduced to avoid a trivial solution depleting the battery. Generally, HEVs are forced to operate in such a way that the battery charge $\left(E_{b}\right)$ at the end of the cycle keeps the same level than at the beginning, i.e. the HEV operates in charge sustaining mode. For convenience it is introduced as a hard constraint:

$$
\Psi=E_{b}(T)-E_{b, 0}=0
$$

where $E_{b, 0}$ is the initial battery energy level, which is a known quantity. Accordingly, the costate terminal constraint from (18) is transformed into:

$$
\lambda(T)=\left.\nu \frac{\partial \Psi}{\partial E_{b}}\right|_{t=T}=\nu
$$

Finally, the costate equations at (17) are particularized as:

$$
\dot{\lambda}(t)=-\frac{\partial H}{\partial E_{b}}=0
$$

where $\dot{\lambda}$ is zero since no term in Hamiltonian explicitly depends on the state $E_{b}$ (neither $L$ nor $f$ ). Therefore, $\lambda$ is constant over the time and equals the unknown $\nu$ according to (23).

Therefore, the energy management problem consists on finding the appropriate set of control actuations that verifies (15) for the Hamiltonian at (21). Necessary conditions for optimality, (22)-(24), must be also fulfilled.

An important remark is that, while it is pretty easy to introduce constraints on the control set (in the form of $u \in \mathcal{U}$ ) by limiting the seeking range for (15), state constraints are hard to include in PMP formulation, so constraints such as (12) have been deliberately obviated. Although usually battery energy level rarely reaches the battery capacity limits in a charge-sustaining non-plugin HEV, in some situations states constraints might be necessary to avoid boundaries violation. A typical workaround is to define an arbitrary penalization function weighting the costates to pull states and keep them away from constraint limits. The result is, of course, suboptimal but feasible. This technique has been extensively discussed in the literature (the interested reader may address to [14], [25] or [31]) but it is out of the scope of this work.

\section{Analytical solution}

The presented analytical solution to the energy management problem consists of an explicit application of PMP, whose basic formulation has been detailed in the previous section. This solution is derived from the necessary conditions for optimality stated in (22)-(24) to extract an analytical expression for the optimal trajectory of the control variable $\left(P_{g}\right)$. Note that although PMP is defined for continuous and differentiable variables as needed by the aforementioned derivatives, the current Hamiltonian is a piecewise function with a jump discontinuity. However, since Hamiltonian derivative in (24) is null in this case and it is still possible to find a minimum for the Hamiltonian according to (15), a relaxation in the continuity requisite is conducted with no impact on the formulation nor the optimality of results.

\subsection{Optimal operation of the generator set}

The minimization of the Hamiltonian with respect to the control actuation $\left(P_{g}\right)$ could be approached by finding the minimum of the two members $\left(H_{1}, H_{2}\right)$ of the piecewise function (21) and, then, selecting the minimum among those two candidates. The minimum of the first member, $H_{1}^{*}$, may be calculated applying first and second derivatives:

$$
\begin{gathered}
\frac{\partial H_{1}}{\partial P_{g}}=a-\frac{\nu V_{o c}}{\sqrt{V_{o c}^{2}+4 R\left(P_{g}-P_{m}\right)}}=0 \\
\frac{\partial^{2} H_{1}}{\partial P_{g}^{2}}=\frac{2 R \nu V_{o c}}{\sqrt{\left[V_{o c}^{2}+4 R\left(P_{g}-P_{m}\right)\right]^{3}}}>0
\end{gathered}
$$

where $\lambda$ has been replaced by a constant $\nu$ according to (23) and (24). The minimum corresponds to:

$$
P_{g, 1}^{*}=P_{m}+\frac{V_{o c}^{2}}{4 R}\left(\frac{\nu^{2}}{a^{2}}-1\right)
$$

and the value of the Hamiltonian at that minimum is:

$$
H_{1}^{*}=P_{f, i d l e}+a P_{m}+\frac{V_{o c}^{2}}{2 R} \nu-\frac{V_{o c}^{2}}{4 R a} \nu^{2}-\frac{a V_{o c}^{2}}{4 R}
$$

which is the first minimum candidate for the Hamiltonian.

The minimum for the second member, $H_{2}^{*}$, is trivial since the expression does not depend on $P_{g}$. Therefore, the corresponding candidate for Hamiltonian's minimum is:

$$
H_{2}^{*}=\frac{\nu V_{o c}}{2 R}\left(V_{o c}-\sqrt{V_{o c}^{2}-4 R P_{m}}\right)
$$

The minimum among candidates $H_{1}^{*}$ and $H_{2}^{*}$ might be calculated by stating the inequality expression:

$$
H_{1}^{*}<H_{2}^{*}
$$

which may be solved resulting in: 


$$
\begin{gathered}
\nu>\frac{a \sqrt{V_{o c}^{2}-4 R P_{m}}+2 \sqrt{R P_{f, i d l e} a}}{V_{o c}} \\
\nu<\frac{a \sqrt{V_{o c}^{2}-4 R P_{m}}-2 \sqrt{R P_{f, i d l e} a}}{V_{o c}}
\end{gathered}
$$

These expressions give the regions where $H_{1}^{*}$ is the absolute minimum of the piecewise Hamiltonian. Otherwise, $H_{2}^{*}$ is the minimum. A singularity may occur when $\nu$ equals any of the right side term of the above equations. In this case, both minimum candidates adopt the same value and, therefore, there are two optima. It can be argued that for a HEV it might be more attractive to shut down the engine whenever is possible to minimize noise, vibrations and wear (so the preference would be selecting $H_{2}^{*}$ in the singularity), but other criteria may be included in the form of additional constraints such as minimization of number of on/off switches, maximization of after-treatment efficiency (preserving a high temperature), etc. In any case, the Hamiltonian as defined in (21) still verifies the necessary condition from (15), so optimality is guaranteed. Therefore, authors decided to give preference to $H_{2}^{*}$ in the singularity to keep the formulation simple and clear.

Additionally to the necessary conditions for optimality, the HEV introduces several constraints in the intermediate variables due to limitations of powertrain machines that must be considered to reach a physically feasible solution. The first limitation is the admissible operation range of the generator set $\left(P_{g}\right)$, which is introduced as a constraint to the space of permitted control actuations $\mathcal{U}$ as early stated in section 2.2 , which limits the generator set power output between 0 and $P_{g, \max }=50 \mathrm{~kW}$. This also constrains the minimum of $(27)$ so that $P_{g, 1}^{*}>0 \mathrm{~kW}$ and $P_{g, 1}^{*} \leq P_{g, \text { max }} \mathrm{kW}$. Accordingly, two new constraints are introduced to the region where the candidate $H_{1}^{*}$ is valid. For the upper bound this constraint is expressed as:

$$
\nu \leq a \sqrt{1-\frac{4 R}{V_{o c}^{2}}\left(P_{m}-P_{g, \max }\right)}
$$

Similarly, the lower bound is expressed as:

$$
\nu>a \sqrt{1-\frac{4 R}{V_{o c}^{2}} P_{m}}
$$

resulting that among constraints in (31) and (32) only the first one is active, i.e. the region where candidate $H_{1}^{*}$ is the minimum is reduced to the first of both.

The second powertrain limitation is the operation capability of the battery, which is restricted in the power output (and current) by its internal resistance $R$ according to (7). Using (8), the constraint results in:

$$
P_{m} \leq \frac{V_{o c}^{2}}{4 R}+P_{g}
$$

for the candidate $H_{1}^{*}$. It can be clearly seen that introducing the value of $P_{g, 1}^{*}$ in the above equation, the inequality is always satisfied, meaning that this constraint is not active.
Additionally, for the candidate $H_{2}^{*}$, the battery limitation introduces a similar constraint:

$$
P_{m} \leq \frac{V_{o c}^{2}}{4 R}
$$

To clarify the present set of constraints at the problem, the figure 3 represents all the above constraints for both minimum candidates, $H_{1}^{*}$ (left plot, in red color) and $H_{2}^{*}$ (center plot, in blue color), for different values of motor power $P_{m}$ (x-axis) and $\nu$ (y-axis). On the candidate $H_{1}^{*}$ side, as it has been previously stated, it is clear that only one of the two regions where $H_{1}^{*}$ is the minimum, expressed in (31) and (32), is feasible due to constraint (34). On the candidate $H_{2}^{*}$ side, the feasible region is bounded by constraints (31) and (36). As it may be appreciated, both $H_{1}^{*}$ and $H_{2}^{*}$ regions are in practice constrained just by these last two equations.

Right plot in figure 3 denotes the combination of both Hamiltonians and the regions where each one is the minimum. Note that there are some particularities: $H_{2}^{*}$ is the minimum even below (31) since it is the only feasible Hamiltonian as $H_{1}^{*}$ is constrained by (34). $H_{1}^{*}$ is the minimum even over (33) as long as $H_{2}^{*}$ is not feasible due to (36). However, in this last region, $H_{1}^{*}$ is saturated because of (33) (the generator set delivers the maximum power output). Therefore, they can be expressed as a piecewise function:

$$
\widehat{P}_{m}= \begin{cases}\frac{V_{o c}^{2}}{4 R}-\frac{P_{f, i d l e}}{a}+ & \\ \frac{V_{o c} \sqrt{R P_{f, i d l e} a}}{R a^{2}} \nu-\frac{V_{o c}^{2}}{4 R a^{2}} \nu^{2} & \\ \frac{V_{o c}^{2}}{4 R} & \nu<\widehat{\nu}\end{cases}
$$

where $\widehat{\nu}$ is:

$$
\widehat{\nu}=\frac{2 \sqrt{R P_{f, i d l e} a}}{V_{o c}}
$$

Accordingly, the candidate $H_{1}^{*}$ is the minimum for any $P_{m}>\widehat{P}_{m}$ while otherwise $H_{2}^{*}$ is the minimum. Therefore, the control actuation $P_{g}^{*}$ that minimizes the Hamiltonian satisfying (15) is:

$$
P_{g}^{*}= \begin{cases}P_{g, 1}^{*} & P_{m}>\widehat{P}_{m} \\ 0 & P_{m} \leq \widehat{P}_{m}\end{cases}
$$

This control policy is the optimal set of actuations as it verifies all the PMP necessary conditions for optimality (as discussed in the last two sections). It is graphically represented in a $P_{m}$ vs $\nu$ plot in figure 4 .

\subsection{On the determination of $\nu$}

The value of $\nu$ must be selected such that the terminal constraint (22) is fulfilled. Numerical methods are available at literature, such that the shooting technique in [35] or the $\lambda$-plots method in [32]. However, the analytical control policy obtained in the previous section permits to explicitly find the exact value of $\nu$ in a straightforward fashion. 

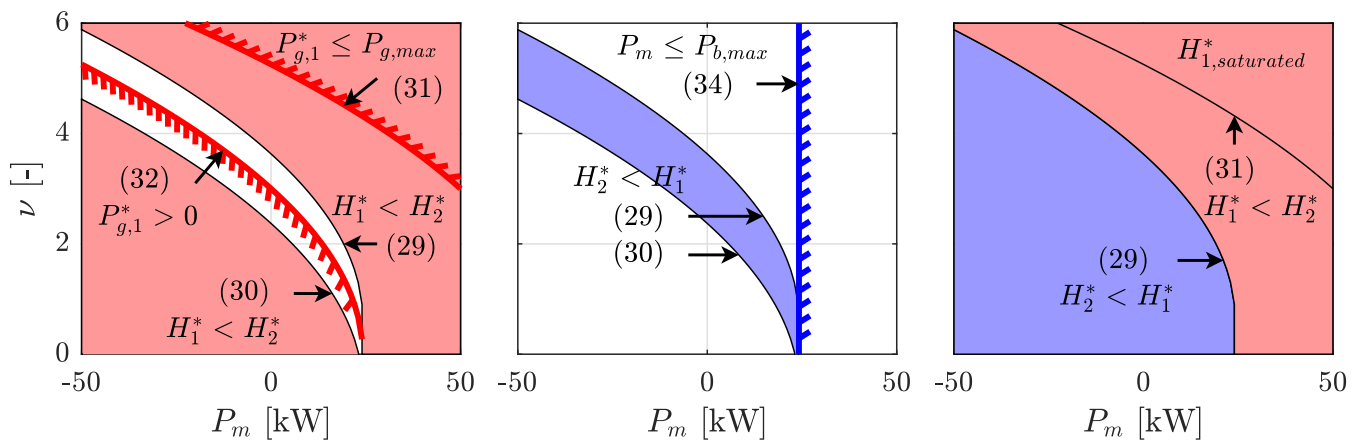

Figure 3: Graphical representation of both candidates for minimum, $H_{1}^{*}$ (left) and $H_{2}^{*}$ (center). Solid colors represent regions where one candidate is the minimum; hatched lines represent constraints (corresponding equations are indicated). Combining both candidates and constraints results in the right plot where active regions are shown for $H_{1}^{*}$ (red) and $H_{2}^{*}$ (blue).

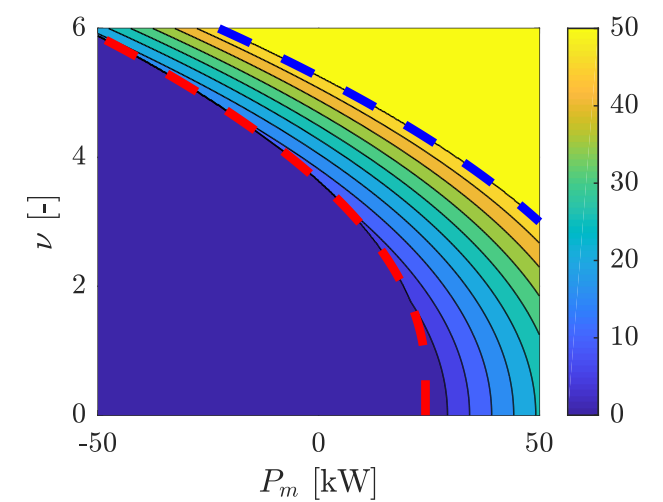

Figure 4: Representation of optimal set of actuations $P_{g}^{*}$ for different levels of traction requirements $\left(P_{m}\right)$ and parameter $\nu$. The red dashed line is the generator set disconnection limit, (37), and the blue dashed line is the generator set maximum power constraint, (33).

Reviewing the steps performed so far, first, (23) shown that $\lambda(T)=\nu$ and, second, (24) proved that $\lambda$ is constant and therefore equals the constant value $\nu$. Thereby, the parameter $\nu$ must be selected such that (22) is fulfilled. Based on the EMP solution, this last equation may be rewritten as:

$$
\begin{array}{r}
\Psi=\int_{0}^{T} f d t+E_{b}(0)-E_{b, 0}=\int_{0}^{T} f d t= \\
\int_{0}^{T} \frac{V_{o c}}{2 R}\left(V_{o c}-\sqrt{V_{o c}^{2}-4 R P_{b}}\right) d t=0
\end{array}
$$

since $\int_{0}^{T} f d t=E_{b}(T)-E_{b}(0)$ according to $(20)$ and $E_{b}(T)-E_{b}(0)=0$. Note that $P_{b}$ is a piecewise function as defined in (8) and (39):

$$
P_{b}(t)= \begin{cases}P_{m}(t)-P_{g, 1}^{*} & P_{m}>\widehat{P}_{m} \\ P_{m}(t) & P_{m} \leq \widehat{P}_{m}\end{cases}
$$

The idea is to integrate $\Psi$ as a function of $\nu\left(P_{g, 1}^{*}\right.$ contains $\nu$ ) and then solve the resulting equation for $\nu$. The main issue when addressing this integral equation is the fact that $P_{b}$ is a piecewise function. In order to avoid this issue, a workaround is to split this integral equation into the sum of two integrals, one where only the first member of the piecewise function applies, and another where only the second member appears. To do so, the proposed method is to separate $P_{m}(t)$ trajectory into $n$ sections $\left\{P_{m, 1}, \cdots, P_{m, n}\right\}$ at times $\left\{t_{1}, \cdots, t_{n-1}\right\}$ such that each part is completely over or under $\widehat{P}_{m}$. Due to continuity reasons, $P_{m}$ sections over and under $\widehat{P}_{m}$ will obviously alternate in time:

$$
\left\{\begin{array}{l}
P_{m, 2 i-1}>\widehat{P}_{m} \\
P_{m, 2 i} \leq \widehat{P}_{m}
\end{array} \quad \forall i \in\left[1, \frac{n}{2}\right]\right.
$$

In the above expression it is assumed that $P_{m, 1}>\widehat{P}_{m}$ and $\frac{n}{2} \in \mathbb{Z}$ for clarity, but the opposite also applies following a similar methodology. Thus, sections with odd subscripts are over $\widehat{P}_{m}$ while sections with even subscripts are under it. Now, $P_{m}(t)$ trajectory may be rearranged into a new $\widetilde{P}_{m}(t)$ trajectory with all odd subscripts first:

$$
\begin{gathered}
\widetilde{P}_{m}=\left[P_{m, 1}, P_{m, 3}, \cdots, P_{m, n-1},\right. \\
\left.P_{m, 2}, P_{m, 4}, \cdots, P_{m, n}\right]
\end{gathered}
$$

where it is clear that the first $\frac{n}{2}$ members are completely above $\widehat{P}_{m}$ and the remaining are below it. The time instant where $\widetilde{P}_{m}$ moves from over to under $\widehat{P}_{m}$, called $\widetilde{T}$, can be easily calculated by summing up the durations of the odd sections of $P_{m}$ :

$$
\widetilde{T}=\sum_{i=1}^{n / 2}\left[t_{2 i-1}-t_{2 i}\right]
$$

Finally, the integral form of $\Psi$ from (40) can be rewritten in terms of $\widetilde{P}_{m}$ :

$$
\begin{aligned}
\Psi= & \int_{0}^{\widetilde{T}} \frac{V_{o c}}{2 R}\left(V_{o c}-\sqrt{V_{o c}^{2}-4 R\left[\widetilde{P}_{m}(t)-P_{g, 1}^{*}\right]}\right) d t+ \\
& \int_{\widetilde{T}}^{T} \frac{V_{o c}}{2 R}\left(V_{o c}-\sqrt{V_{o c}^{2}-4 R \widetilde{P}_{m}(t)}\right) d t
\end{aligned}
$$

Operating and simplifying, $\Psi$ results: 


$$
\Psi=\frac{V_{o c}}{2 R}\left[V_{o c}\left(T-\frac{\nu}{a} \widetilde{T}\right)-\int_{\widetilde{T}}^{T} \sqrt{V_{o c}^{2}-4 R \widetilde{P}_{m}(t)} d t\right]
$$

Then, solving $\Psi=0$ for $\nu$ brings the following expression:

$$
\nu=\frac{a T}{\widetilde{T}}-\frac{a}{V_{o c} \widetilde{T}} \int_{\widetilde{T}}^{T} \sqrt{V_{o c}^{2}-4 R \widetilde{P}_{m}(t)} d t
$$

Note that the above expression losses its time dependency as $\widetilde{P}_{m}$ is integrated, substituting $t$ for the integration limits $\widetilde{T}$ and $T$. As long as the trajectory $\widetilde{P}_{m}$ is a rearrangement of the actual motor power trajectory $P_{m}$ according to its value-see (43)-, any other $P_{m}$ trajectory with the same power distribution would lead to the same integrated value-the order of the discrete values in $P_{m}$ is not relevant. In fact, $P_{m}$ can be replaced with its probability distribution for practical reasons delivering the same results.

The existence of an explicit solution for the integral in the above equation depends on the expression given for $\widetilde{P}_{m}(t)$, not only because of the explicit presence of this variable but also because $\widetilde{T}$ is a function that depends on the threshold $\widehat{P}_{m}$ which, according to (37), is a function of $\nu$. However, if no explicit solution exists, it can still be solved by iterating in (37) and (47).

\subsection{Example of analytical optimization}

To clarify the above method, an example of the optimization of a series HEV operation along a simple trajectory is provided below. The $P_{m}(t)$ trajectory is depicted at the left plot in figure 5 and consists of a ramp with a constant decreasing rate, from positive traction to energy recovery, that follows the expression:

$$
P_{m}(t)=P_{m}^{0}-k t
$$

where in this case $P_{m}^{0}=40 \mathrm{~kW}, k=0.5 \mathrm{~kW} / \mathrm{s}$, and $t \in$ $[0,120]$ seconds. This trajectory is deliberately selected to be such a simple case to keep formulation clear and understandable. Anyhow, it can be interpreted as a driver approaching a full stop, slowly releasing the pedal from positive to negative traction requirements.

First the appropriate value of $\nu$ that fulfills the terminal constraint must be found. To do so, $P_{m}(t)$ must be rearranged according to (42) and (43). The trajectory in this case is monotonically decreasing, so there is only one section of $P_{m}$ over $\widehat{P}_{m}$ and another one below it. Therefore the rearrangement results in $\widetilde{P}_{m}(t)=P_{m}(t)$. Introducing this $\widetilde{P}_{m}$ in (47) brings an explicit expression for $\nu$ :

$$
\begin{aligned}
\nu=a & \left(\frac { 1 } { 6 R k } \left[\left(V_{o c}^{2}-4 R k T\right)^{3 / 2}-\right.\right. \\
& \left.\left.\left(V_{o c}^{2}-4 R k \widetilde{T}\right)^{3 / 2}\right]+\frac{T}{\widetilde{T}}\right)
\end{aligned}
$$

$\begin{array}{lr}\text { Battery open circuit voltage, } V_{o c}[\mathrm{~V}] & 220 \\ \text { Battery internal resistance, } R[\mathrm{Ohm}] & 0.5 \\ \text { Fuel consumption at idle, } P_{f, \text { idle }}[\mathrm{kW}] & 3.2 \\ \text { Fuel consumption coefficient, } a[-] & 3\end{array}$

Table 2: Powertrain parameters and their values.

Note that in the above equation the integral from (47) has been solved for $\widetilde{P}_{m}$.

Next, $\widetilde{T}$ should be calculated (total time $P_{m}$ stands over $\left.\widehat{P}_{m}\right)$. Since $P_{m}$ is a monotonic function, $\widetilde{T}$ can be easily solved as:

$$
\widetilde{T}=\frac{P_{m}^{0}-\widehat{P}_{m}}{k}
$$

Substituting $\widehat{P}_{m}$ with its analytic expression from (37) leads to:

$$
\widetilde{T}= \begin{cases}\frac{V_{o c}^{2}}{4 R a^{2} k} \nu^{2}-\frac{V_{o c} \sqrt{R P_{f, \text { idle }} a}}{R a^{2} k} \nu+ & \\ \frac{4 R P_{f, \text { idle }}+4 R a P_{m}^{0}-a V_{o c}^{2}}{4 R a k} & \nu \geq \widehat{\nu} \\ \frac{4 R P_{m}^{0}-V_{o c}^{2}}{4 R k} & \nu<\widehat{\nu}\end{cases}
$$

At this point there are two unknowns $-\widetilde{T}$ and $\nu$, and two equations-(49) and (51). The idea is to solve $\nu$ explicitly, but unfortunately (49) implies a fifth degree polynomial in terms of $\widetilde{T}$, so a general explicit solution for $\nu$ does not exist. However, it is still possible to analytically reach a solution for specific values of the parameters. In this case, the parameters at table 2 have resulted in a value of $\nu=2.776$.

$\widetilde{T}$ for the above value of $\nu$ is 56.4 seconds, and the threshold $\widehat{P}_{m}$ results in a value of $11.8 \mathrm{~kW}$. Therefore, the optimal trajectory for the generator set is:

$$
P_{g}^{*}(t)= \begin{cases}36.5-0.5 t & t<56.4 \mathrm{~s} \\ 0 & t \geq 56.4 \mathrm{~s}\end{cases}
$$

This optimal trajectory $P_{g}^{*}(t)$ as well as the evolution of battery's SoC (with initial SoC level at 40\%) are represented in figure 5 where it can be appreciated that the terminal constraint (same $\mathrm{SoC}$ at the end than at the beginning) is precisely fulfilled.

Despite this case showed a pretty simple $P_{m}(t)$ trajectory for clearness, more complex evolutions can be considered following the same methodology, even if they are not explicitly integrable (calculating $\nu$ by numerical iteration). Of course if $P_{m}(t)$ is not integrable this method is not as straightforward as the fully explicit approach, but it is still much more efficient than the common shooting technique since this only involves iteration in a single equation instead of a full trajectory simulation. 

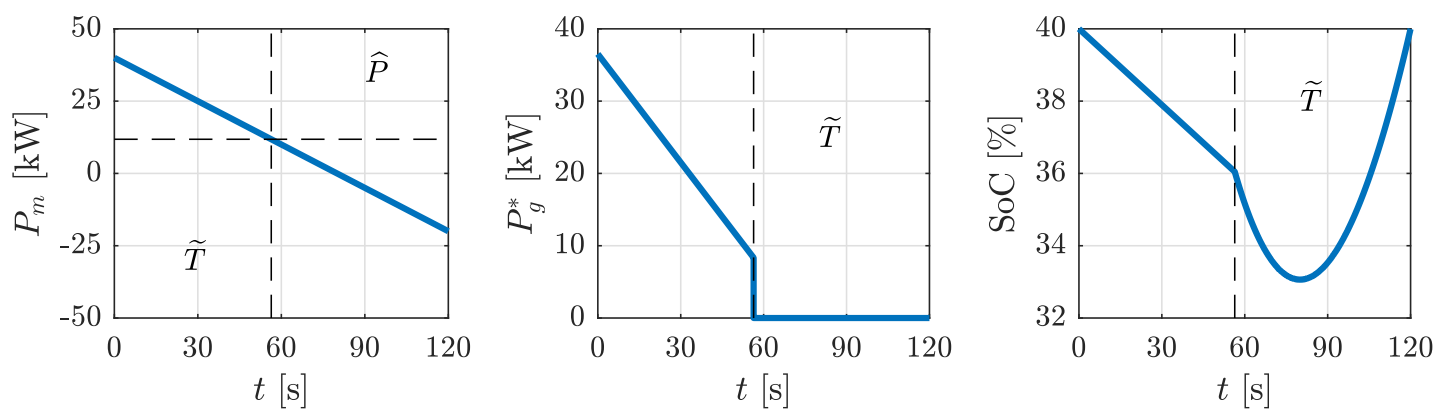

Figure 5: Trajectory and optimization solution for an example case of 120 seconds. The left plot shows the $P_{m}$ trajectory, the middle plot shows the optimized generator set power output, and the right plot shows the variations on battery's SoC throughout the example with an initial level of $40 \%$.

\begin{tabular}{lr}
\hline Mass [kg] & 1300 \\
Cd [-] & 0.3 \\
Reference area $\left[\mathrm{m}^{2}\right]$ & 2.0 \\
Rolling resistance coefficient [-] & 0.01 \\
Nominal motor efficiency [-] & 0.85 \\
Battery open circuit voltage [V] & 220 \\
Battery internal resistance [Ohm] & 0.5 \\
Battery capacity $[\mathrm{Ah}]$ & 6.9 \\
\hline
\end{tabular}

Table 3: Main characteristics of simulated series HEV.

\section{Case study}

\subsection{Optimal control policy}

An analytical solution to the EMP in a series HEV has been stated in the previous section, summarized in (39). This equation indicates the amount of power the generator set must deliver throughout time, $P_{g}^{*}(t)$, to minimize fuel consumption, being only a function on the instantaneous traction motor power required to follow a driving cycle, $P_{m}$.

The aim of this section is to benchmark and validate the optimality of this analytical solution to the EMP on a real driving trajectory compared to the most common optimization methods in literature: dynamic programming (DP) and numerical Pontryagin minimum principle (PMP).

The benchmark driving trajectory consists of 1,122 seconds of urban driving, recorded at public roads with a light duty vehicle. The cycle presents several accelerations, a couple of strong brakings and some stops of different durations, representing a wide variety of driving situations. Figure 6 illustrates this driving trajectory and some of its statistics.

The main characteristics of the vehicle used for simulations are summarized at table 3 . The utilized powertrain parameters are specified in table 2 . The initial SoC level is arbitrarily set to $30 \%$-actually this level is a consequence of the previous usage of the vehicleand, since it is a charge sustaining HEV, the final SoC constraint is also set to $30 \%$.

First of all, the optimal solution to the EMP has been calculated with Dynamic Programming. DP is based on Bellman's Principle of Optimality to iteratively compute the optimal trajectory among all possibilities from an initial space of feasible solution candidates. In other words, the method globally minimizes the integral cost function $J$ at (9). Theoretically, the solution achieved is the global optimum to the problem since roughly all possibilities are checked with no further assumptions; however, the resulting solution is restricted to a discrete grid of candidates, which could lead to a suboptimal solution for a continuous problem.

Following, the EMP has been also solved with numerical PMP. As [10] states, PMP is equivalent to DP when the necessary conditions for the global optimality are met. Thus, the global optimization problem approached with DP is converted into a local problem where the Hamiltonian, represented by (21), is minimized at each time instant. The costate $\lambda$ is tuned by shooting technique in order to satisfy the terminal state constraint at (22).

Finally the analytical policy presented in previous sections of this work is evaluated. The parameter $\nu$ is calculated with (37) and (47) by means of numerical iteration. Then the EMP solution is just constructed substituting values at (39).

All three methods have been implemented and simulated with a backwards series HEV model as described in section 2.1. The simulation results are shown in figure 7 where the optimal control policy may be appreciated, namely the control actuation $\left(P_{g}\right)$ and the battery state of charge, SoC, which is proportional to the state of the system, $E_{b}$. Additionally, figure 8 shows the correlation between analytical method and DP and PMP fuel consumption. The total fuel consumption, which is the minimization target, is $3.44,3.45$ and $3.45 \mathrm{~L} / 100 \mathrm{~km}$ for DP, PMP and analytic methods respectively. The computation time that each method takes to find the optimal solution is pretty interesting, especially if targeting for an online implementation where calculation time is limited. Figure 9 summarizes the necessary time to reach the EMP solution, where it can be appreciated that analytical method is the fastest ( 0.03 seconds), followed by numerical PMP ( 0.73 seconds) and DP (76.4 seconds), where the "curse of dimensionality" strongly penalizes the computation time.

According to the results it is clear that the calculated optimal control policies are quite the same regardless 

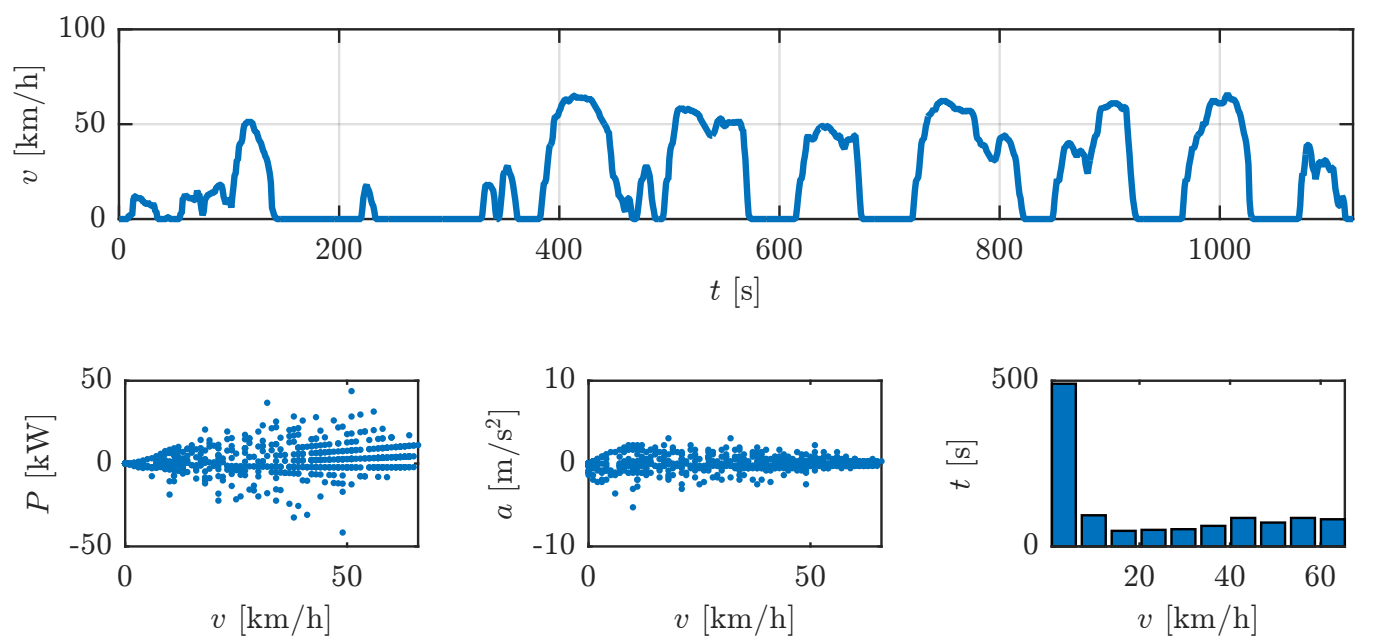

Figure 6: Benchmarking driving trajectory. The upper plot depicts the speed of the vehicle along 1,122 seconds. The bottom plots show, from left to right, the distribution over the vehicle speed of: power requirement for a light duty vehicle, acceleration and time dedicated during cycle
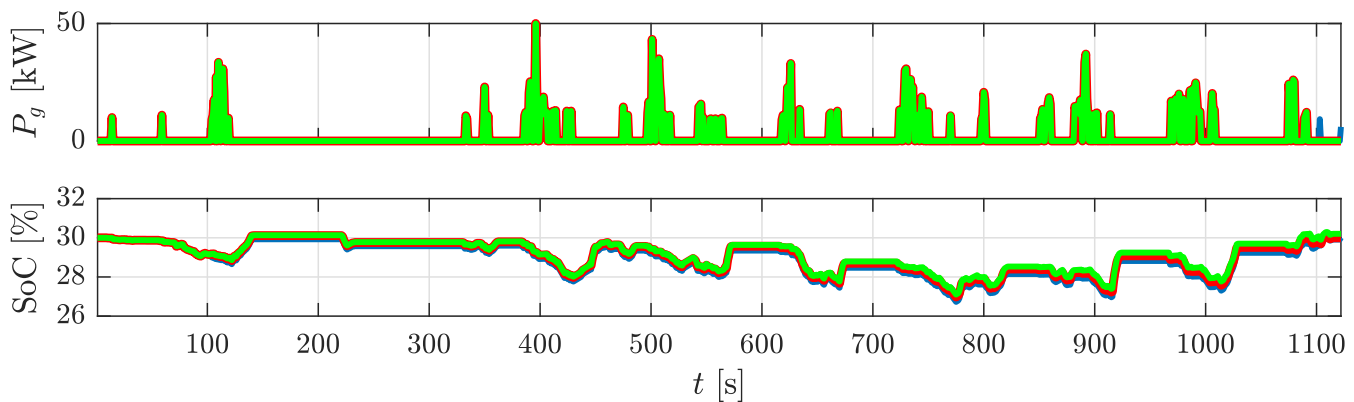

Figure 7: DP (blue), PMP (red) and analytical method (green) results. The upper plot corresponds to the control action, $P_{g}$, and the bottom plot to the state, battery SoC

of the method. On one hand, numerical PMP and the analytical method result in an almost identical solution, as the analytical method is an explicit formulation of the PMP applied to a series HEV. Differences are only due to numerical issues at integration and costate seeking in the numerical PMP. On the other hand, in this case the DP method is equivalent to PMP as, according to [10], the necessary conditions for optimality are met; any difference is just due to the discretization of control and state.

\subsection{Identification of the engine opera- tion area}

The existence of an explicit expression for the optimal control policy allows performing some easy yet useful calculations that reveals key parameters of the operation of the HEV. Probably one of the most interesting analysis is to identify the region of the engine or of the generator set where the powertrain operates the most for a type of driving cycle or a set of cycles.

One of the key points of the explicit solution is that the presented EMP ultimately depends on the power requirements of the cycle but not on the time reference. According to this, it is possible to replace the power

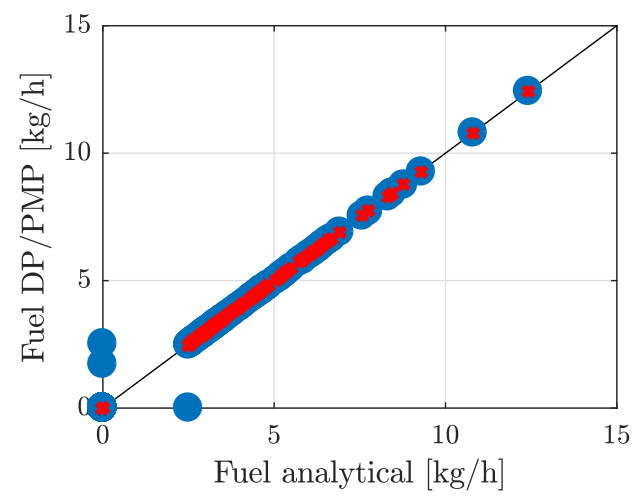

Figure 8: DP (blue) and PMP (red) fuel consumption correlation with respect to analytical method. The $\mathrm{x}$-axis corresponds to analytical method and $\mathrm{y}$-axis to DP/PMP fuel consumption

requirements trajectory of interest with its probability distribution. The use of a probability distribution allows to extract conclusions about the optimal operation of the powertrain in a more compact and general form, similarly to [23] and [35].

The methodology is quite straightforward to analyze a particular driving cycle whose probability distribu- 


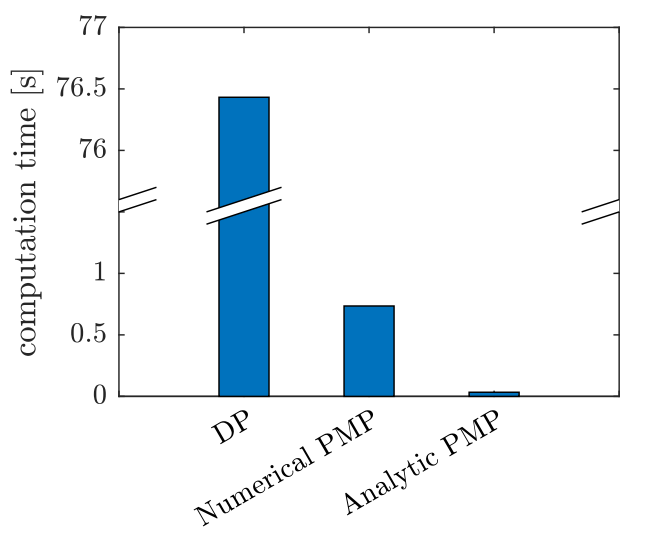

Figure 9: Computation time of optimization methods at the example driving cycle.

tion, $\mathrm{PD}$, of power requirements is $\operatorname{Pr}=\operatorname{pdf}_{P_{m}}\left(P_{m}\right)$ and its cumulative distribution, $\mathrm{CD}$, is $\operatorname{cdf}_{P_{m}}\left(P_{m}\right)=$ $\int \operatorname{pdf}_{P_{m}} d P_{m}$, which might be both explicit expressions or discrete sets of values. Following the steps from 3.2 it is possible to determine the value of $\nu$, taking into account that in this case $\widetilde{T} / T=\operatorname{cdf}_{P_{m}}\left(\widehat{P}_{m}\right)$ and $\widetilde{P}_{m}=\operatorname{cdf}_{P_{m}}^{-1}$. Then, the explicit solution to the EMP may be obtained by applying (39). The PD of the generator set operation is, therefore:

$$
\operatorname{pdf}_{P_{g}}\left(P_{g}\right)= \begin{cases}\operatorname{cdf}_{P_{m}}\left(\widehat{P}_{m}\right) & P_{g}=0 \\ 0 & 0<P_{g} \leq \widehat{P}_{g} \\ \operatorname{pdf}_{P_{m}}\left(P_{g}-\right. & \widehat{P}_{g}<P_{g} \\ \left.\frac{V_{o c}^{2}}{4 R}\left(\frac{\nu^{2}}{a^{2}}-1\right)\right) & \end{cases}
$$

where $\widehat{P}_{g}$ is:

$$
\widehat{P}_{g}=\widehat{P}_{m}+\frac{V_{o c}^{2}}{4 R}\left(\frac{\nu^{2}}{a^{2}}-1\right)
$$

The above methodology has been applied to a set of diverse real driving cycles of urban and highway recordings from non-professional drivers. On one hand the $\mathrm{PD}$ of the power requirements of the cycle have been calculated for each cycle and then (53) has been applied to find out the region of the generator set that is operated the most. On the other hand the same results have been reached by optimizing the HEV operation with DP and, then, calculating the PD of the solution for benchmarking purposes. PD's may be appreciated in figure 10.

According to the results it is clear that close estimations of the operation range of the generator set could be obtained with both explicit method or DP optimization. However, the explicit approach as presented in (53) shows two main advantages over DP or any other trajectory optimization algorithm: first, only the PD of the driving cycle is required to apply the explicit method permitting to analyze the generator set operation for different driving styles rather than for particular driving cycles and, second, the computational cost is nearly zero as long as only a couple of algebraic equations must be calculated (DP may take from minutes to hours to optimize a single driving cycle) making easier to quickly analyze a vast set of different driving styles.

\section{Conclusions}

This work presents an analytical solution to the EMP in a series HEV. This method is an explicit implementation of the PMP applied to a generic and validated $\mathrm{HEV}$ model, resulting in an expression that gives the optimal amount of power that the generator set needs to deliver as a function of the instantaneous traction power requirement $P_{m}$.

The explicit calculation of the Lagrangian multipliers associated to the PMP is also discussed in this study. According to the necessary conditions for optimality, an analytical expression to calculate Lagrangian multipliers is obtained in an integral form. An iterative methodology to solve this integral equation is provided for cases where a fully explicit solution does not exist.

The optimization of a driving cycle with the analytical approach as well as DP and numerical PMP is provided as an example. All three algorithms shown nearly the same results while the analytical method was the fastest by far.

The availability of an explicit expression for the solution of the EMP makes this method specially interesting for optimal solution analysis. It is straightforward to analyze the impact on the optimal control (generator set operation, fuel consumption, etc.) when changing any of the vehicle parameters. As an example, the analysis of the operation region of the generator set as been discussed and applied to some driving cycles. This methodology has been shown to be much more computationally efficient and general than reaching the same results with DP.

It must be remarked that the applicability of this analytical methodology to other hybrid powertrain architectures will be up to the complexity of the model and, especially, to its non-time dependency; otherwise, the resulting control algorithm will be cycle-dependent.

In conclusion, the compactness, small computational burden and simplicity of the explicit optimal solution for such a complex EMP makes it particularly interesting to assess studies with big sets of driving styles or to implement on a real vehicle with a modest hardware. Future works might be addressed to analyze and implement this method as an optimal energy management controller for on-board applications and to explore its applicability to other hybrid powertrain architectures.

\section{References}

[1] A Sciarretta and L Guzzella. "Control of Hybrid Electric Vehicles". In: IEEE Control System Magazine 27.2 (2007), pp. 60-70. 

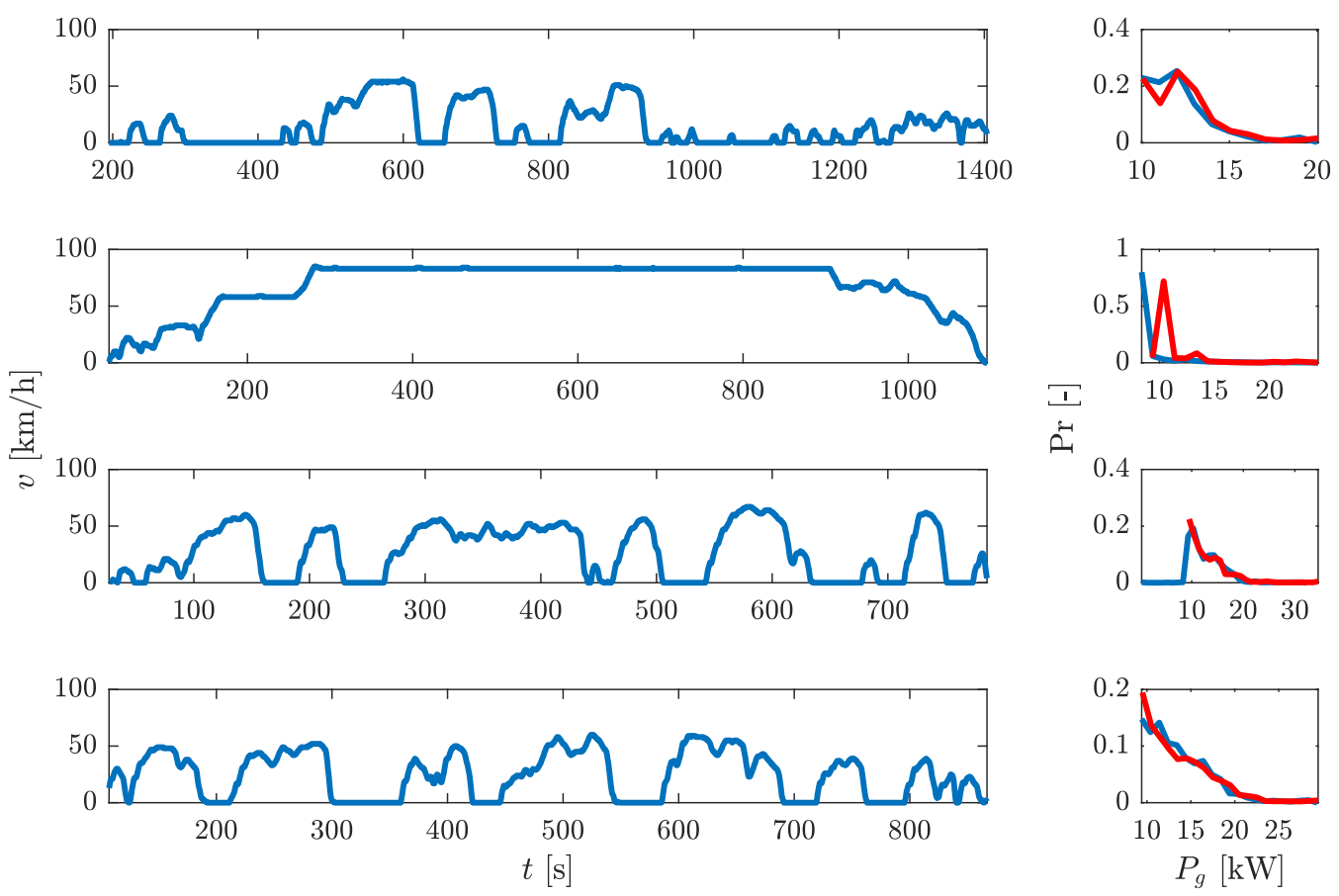

Figure 10: Operation region of the generator set when optimally controlled in various real driving cycles. Left plots show speed profile of the driving cycles. Right plots show the probability distribution of the generator set operation as calculated with the explicit expression presented in this work (blue) and with the common DP optimization (red).

[2] L Serrao, S Onori, and G Rizzoni. "A Comparative Analysis of Energy Management Strategies for Hybrid Electric Vehicles". In: Journal of Dynamic Systems, Measurement, and Control 133 (2011), pp. 1-9.

[3] S Stockar et al. "Energy-Optimal Control of Plugin Hybrid Electric Vehicles for Real-World Driving Cycles". In: IEEE Transactions on Vehicular Technology 60.7 (2011), pp. 2949-2962.

[4] S J Moura et al. "A Stochastic Optimal Control Approach for Power Management in Plug-In Hybrid Electric Vehicles". In: IEEE Transactions on Control Systems Technology 19.3 (2011), pp. 545555 .

[5] H Mosbech. "Optimal control of a hybrid vehicle". In: Proceedings of the International Symposium on Automotive Technology \& Automation. 1980, pp. 303-320.

[6] O Sundström, D Ambühl, and L Guzzella. "On Implementation of Dynamic Programming for Optimal Control Problems with Final State Constraints". In: Oil \& Gas Science and Technology - Revue d'IFP Energies nouvelles 65.1 (2010), pp. 91-102.

[7] Tobias Nuesch et al. "Optimal energy management and sizing for hybrid electric vehicles considering transient emissions". In: Engine and Powertrain Control, Simulation and Modeling. Vol. 3. 1. 2012 , pp. $278-285$.
[8] Sebastien Delprat et al. "Control strategy optimization for an hybrid parallel powertrain". In: Proceedings of the American Control Conference. Vol. 2. 2001, pp. 1315-1320.

[9] J Bernard et al. "Fuel efficient power management strategy for fuel cell hybrid powertrains". In: Control Engineering Practice 18.4 (2010), pp. 408417.

[10] N Kim, S Cha, and H Peng. "Optimal Control of Hybrid Electric Vehicles Based on Pontryagin's Minimum Principle". In: IEEE Transactions on Control Systems Technology 19.5 (2011), pp. 1279 1287.

[11] Lorenzo Serrao et al. "Open issues in supervisory control of hybrid electric vehicles: a unified approach using optimal control methods". In: Oil \& Gas Science and Technology - Revue d'IFP Energies nouvelles 68.1 (2013), pp. 23-33.

[12] C Guardiola et al. "Insight into the $\mathrm{HEV} / \mathrm{PHEV}$ optimal control solution based on a new tuning method". In: Control Engineering Practice 29 (2014), pp. 247-256.

[13] Guerra Paganelli et al. "Simulation and assessment of power control strategies for a parallel hybrid car". In: Proceedings of the Institution of Mechanical Engineers, Part D: Journal of Automobile Engineering 214.7 (2000), pp. 705-717.

[14] G Paganelli et al. "General Supervisory Control Policy for the Energy Optimization of ChargeSustaining Hybrid Electric Vehicles". In: JSAE Review 22.4 (2001), pp. 511-518. 
[15] Paganelli Rodatz et al. "Optimal power management of an experimental fuel cell / supercapacitorpowered hybrid vehicle". In: Control Engineering Practice 13.1 (2005), pp. 41-53.

[16] L Serrao, S Onori, and G Rizzoni. "ECMS as realization of Pontryagin's minumum principle for HEV control". In: American Control Conference. 2009, pp. 3964-3969.

[17] A. Chasse and A. Sciarretta. "Supervisory control of hybrid powertrains: An experimental benchmark of offline optimization and online energy management". In: Control Engineering Practice 19.11 (2011), pp. 1253-1265.

[18] Alberto Reig and Luigi del Re. "Evaluation of Impact on Pollution of a Linear HEV Optimal Control Strategy by Engine-In-The-Loop Simulation". In: 7th IFAC Symposium on Advances in Automotive Control. Vol. 7. 1. 2013, pp. 29-34.

[19] D Ambühl and Lino Guzzella. "Predictive reference signal generator for hybrid electric vehicles". In: IEEE Transactions on Vehicular Technology 58.9 (2009), pp. 4730-4740.

[20] Lars Johannesson, Stefan Pettersson, and Bo Egardt. "Predictive energy management of a 4QT series-parallel hybrid electric bus". In: Control Engineering Practice 17.12 (2009), pp. 1440-1453.

[21] Francisco Payri et al. "On a stochastic approach of the ECMS method for energy management in hybrid electric vehicles". In: Engine and Powertrain Control, Simulation and Modeling. Vol. 3. 1. 2012, pp. 341-348.

[22] Saida Kermani et al. "Predictive energy management for hybrid vehicle". In: Control Engineering Practice 20.4 (2012), pp. 408-420.

[23] F. Payri et al. "A stochastic method for the energy management in hybrid electric vehicles". In: Control Engineering Practice 29 (2014), pp. 257265.

[24] Antonio Sciarretta, Michael Back, and Lino Guzzella. "Optimal control of parallel hybrid electric vehicles". In: IEEE Transactions on Control Systems Technology 12.3 (2004), pp. 352-363.

[25] C Musardo et al. "A-ECMS: An adaptive algorithm for hybrid Electric Vehicle Energy Management". In: European Journal of Control, Fundamental Issues in Control, Special Issue 11 (2005), pp. 1816-1823.

[26] Jong-Seob Won and Reza Langari. "Intelligent energy management agent for a parallel hybrid vehicle-part I: system architecture and design of the driving situation identification process". In: IEEE Transactions on Vehicular Technology 54.3 (2005), pp. 925-934.

[27] S Onori, L Serrao, and G Rizzoni. "Adaptive equivalent consumption minimization strategy for hybrid electric vehicles". In: Proceedings of the ASME Dynamic Systems and Control Conference. Vol. 1. 2010, pp. 499-505.
[28] Thijs van Keulen et al. "Design, implementation, and experimental validation of optimal power split control for hybrid electric trucks". In: Control Engineering Practice 20.5 (2012), pp. 547-558.

[29] Chan-Chiao Lin, Huei Peng, and J W Grizzle. "A stochastic control strategy for hybrid electric vehicles". In: Proceedings of the American Control Conference. Vol. 5. 2004, pp. 4710-4715.

[30] Daniel F Opila et al. "An energy management controller to optimally trade off fuel economy and drivability for hybrid vehicles". In: IEEE Transactions on Control Systems Technology 20.6 (2012), pp. 1490-1505.

[31] A Chasse, A Sciarretta, and J Chauvin. "Online optimal control of a parallel hybrid with costate adaptation rule". In: Proceedings of the 6th IFAC Symposium: Advances in Automotive Control. 2010, pp. 99-104.

[32] Carlos Guardiola et al. "A new approach to optimally tune the control strategy for hybrid vehicles applications". In: Engine and Powertrain Control, Simulation and Modeling. Vol. 3. 1. 2012, pp. 255261.

[33] Namwook Kim et al. "Optimal control of a plug-in hybrid electric vehicle (PHEV) based on driving patterns". In: International Battery, Hybrid and Fuel Cell Electric Vehicle Symposium. 2009, pp. 19.

[34] C. Zhang and A. Vahid. "Real-time optimal control of plug-in hybrid vehicles with trip preview". In: Proceedings of the American Control Conference. June 2010, pp. 6917-6922.

[35] C. Guardiola et al. "Modelling driving behaviour and its impact on the energy management problem in hybrid electric vehicles". In: International Journal of Computer Mathematics 91.1 (2014), pp. $147-156$.

[36] Anders Fröberg, Erik Hellström, and Lars Nielsen. "Explicit Fuel Optimal Speed Profiles for Heavy Trucks on a Set of Topographic Road Profiles". In: SAE Technical Paper. 2006.

[37] Antonio Sciarretta, Giovanni De Nunzio, and Luis Leon Ojeda. "Optimal Ecodriving Control: Energy-Efficient Driving of Road Vehicles as an Optimal Control Problem". In: IEEE Control Systems 35.5 (2015), pp. 71-90.

[38] Vital van Reeven et al. "Optimal control of engine warmup in hybrid vehicles". In: Oil $\mathscr{E}$ Gas Science and Technology-Revue d'IFP Energies nouvelles 71.1 (2016), pp. 1-18.

[39] Laura Tribioli et al. "A Real Time Energy Management Strategy for Plug-in Hybrid Electric Vehicles based on Optimal Control Theory". In: Energy Procedia 45 (2014), pp. 949-958.

[40] K McDonough et al. "Stochastic dynamic programming control policies for fuel efficient intraffic driving". In: American Control Conference. 2012, pp. 3986-3991. 
[41] Daniel Ambühl et al. "Explicit optimal control policy and its practical application for hybrid electric powertrains". In: Control Engineering Practice 18.12 (2010), pp. 1429-1439.

[42] Edwin Tazelaar, Bram Veenhuizen, Mark Grimminck, et al. "Analytical solution of the energy management for fuel cell hybrid propulsion systems". In: IEEE Transactions on Vehicular Technology 61.5 (2012), pp. 1986-1998.

[43] TH Pham et al. "Analytical solution to energy management guaranteeing battery life for hybrid trucks". In: IEEE Transactions on Vehicular Technology 65.10 (2016), pp. 7956-7971.

[44] Hongwen He, Rui Xiong, and Jinxin Fan. "Evaluation of lithium-ion battery equivalent circuit models for state of charge estimation by an experimental approach". In: Energies 4.4 (2011), pp. 582-598.

[45] L S Pontryagin et al. The mathematical theory of optimal processes. Interscience, New York, 1962.

[46] Arthur E Bryson and Yu-Chi Ho. Applied optimal control: Optimization, estimation, and control. Taylor \& Francis, 1975. 